\title{
Surgical Management of Lower Gastrointestinal Hemorrhage: An Analysis of the ACS NSQIP Database
}

\author{
Laura T. Greco ${ }^{\mathrm{a}, \mathrm{b}}$, Sarah Koller ${ }^{\mathrm{a}}$, Matthew Philp ${ }^{\mathrm{a}}$, \\ Howard Ross ${ }^{a}$
}

\begin{abstract}
Background: Despite advances in diagnostics for lower gastrointestinal bleeding, colorectal resection remains the only option when nonsurgical management fails. This study examines a cohort of patients who underwent surgery for this indication to determine the effect of procedure type on postoperative outcomes.

Methods: We identified all patients who underwent colorectal resection for bleeding in the ACS NSQIP Participant Use Data File and the Procedure Targeted PUF for colectomy from 2012 to 2013. We compared patients who underwent partial versus total colectomy using univariate analyses and multivariable logistic regression.

Results: Of 38,486 colorectal resections performed for bleeding, $85.3 \%$ underwent a partial colectomy and $14.7 \%$ underwent total colectomy. Patients who had total colectomy were more likely to receive more than four units of blood prior to surgery and have operative times longer than $180 \mathrm{~min}$. Patients who had partial colectomy were more likely to have laparoscopic procedures and to have a stoma created during surgery. On univariate analysis, total colectomy was associated with increased risk of postoperative ileus, cardiac and renal complications, and mortality. On multivariate analysis, total colectomy was associated with increased risk of cardiac and renal complications.
\end{abstract}

Conclusion: The most common procedure performed for lower gastrointestinal hemorrhage was partial colectomy.

Keywords: Lower gastrointestinal hemorrhage; Total colectomy; Partial colectomy

\section{Introduction}

In the past 10 years, there has been little new data published

Manuscript accepted for publication November 30, 2016

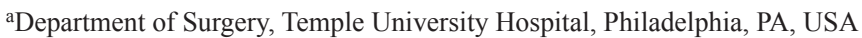
${ }^{b}$ Corresponding Author: Laura Greco, Department of Surgery, Temple University Hospital, 3401 N Broad St., Philadelphia, PA 19144, USA.

Email: Laura.Greco@tuhs.temple.edu

doi: https://doi.org/10.14740/jcs307w regarding surgery for lower gastrointestinal hemorrhage. Despite advances in medical diagnostics, colorectal resection remains the only option when non-surgical management fails. There is little consensus in the literature regarding whether total abdominal colectomy or partial colectomy is superior for surgical treatment of this diagnosis. Several papers have discussed the surgical management of lower gastrointestinal hemorrhage with varying conclusions regarding the benefits of each surgical approach. This study is a retrospective analysis of American College of Surgeons National Surgery Quality Improvement Program (ACS NSQIP) data of patients who have undergone colorectal resection for the diagnosis of lower gastrointestinal hemorrhage. The purpose of this study was to examine a recent cohort of patients who underwent surgery for this indication, given advances in imaging and non-invasive therapeutic modalities. We also sought to explore the effect of procedure type on postoperative outcomes.

\section{Methods}

We identified all patients who underwent colorectal resection for bleeding in both the ACS NSQIP Participant Use Data File (PUF) and the Procedure Targeted PUF for colectomy for the years 2012 - 2013 [1]. We compared patients who underwent partial colectomy to those who underwent total colectomy. Univariate analyses were used to compare the demographics/ co-morbidities and operative characteristics of both groups, and adjusted odds ratios (ORs) and 95\% confidence intervals (CIs) were calculated using multivariable logistic regression.

\section{Results}

A total of 38,486 patients underwent colorectal resections and were included in the database from 2012 to 2013. Of those, 427 procedures were performed for bleeding. The majority were male $(57.4 \%)$ and 65 years of age or older $(68.6 \%)$. About half of surgeries $(49.0 \%)$ were performed emergently. Open procedures $(66.9 \%)$ were more common than laparoscopic procedures. Of the patients, $17.8 \%$ had a stoma created at the time of surgery (Table 1), 85.3\% $(\mathrm{N}=364)$ underwent a partial colectomy, and $14.7 \%(\mathrm{~N}=63)$ underwent total colectomy. Patients who had total colectomy were more likely than those with partial colectomies to have received more than four units of blood prior to surgery $(77.8 \% \mathrm{vs}$. 
Table 1. Demographics of Patients Undergoing Colectomy for Hemorrhage

\begin{tabular}{ll}
\hline Colectomy for indication of hemorrhage \\
\hline Male & $57 \%$ \\
\hline Age > 65 years & $69 \%$ \\
Emergent & $49 \%$ \\
Open surgery & $67 \%$ \\
Stoma created at surgery & $18 \%$ \\
Partial colectomy & $85 \%$ \\
Total colectomy & $17 \%$ \\
\hline
\end{tabular}

$55.5 \%, \mathrm{P}<0.01)$ and to have operative times longer than 180 $\min (42.9 \%$ vs. $23.4 \%, \mathrm{P}<0.01)$. Patients who had partial colectomy were more likely to have undergone laparoscopic procedures $(35.3 \%$ vs. $20.0 \%, \mathrm{P}=0.02)$ and to have a stoma created at the time of surgery $(20.6 \%$ vs. $1.6 \%, \mathrm{P}<0.01)$ (Table 2). On univariate analysis, total colectomy was associated with an increased risk of postoperative ileus, cardiac and renal complications, and mortality (all $\mathrm{P}<0.05$ ), but not with surgical site infection, anastomotic leak, return to the OR, or readmissions. On multivariate analysis, total colectomy was associated with increased risk of cardiac complications (OR: 5.53, 95\% CI: $1.3-22.8$ ) and renal complications (OR: 9.6, 95\% CI: $2.2-43.0)$, but not with ileus $(\mathrm{P}=0.21)$ or mortality $(\mathrm{P}=0.10)$.

\section{Discussion}

A major finding of our review of the NSQIP Database is the preponderance of patients who underwent partial colectomy for the indication of lower gastrointestinal hemorrhage. This may be due to recent improvements in localization of the source of hemorrhage in the form of CTA and angiography. Hoedema and Luchtefeld noted that approximately 10-25\% of patients presenting with lower gastrointestinal hemorrhage had surgical resection, and that total abdominal colectomy was noted to be the operation of choice for non-localized lower gastrointestinal hemorrhage over partial colectomy, due to decreased rates of recurrent bleeding (less than $4 \%$ vs. $14-42 \%$ ) or re-bleeding with partial colectomy.

Multiple previous papers, including one by Schuetz and Jauch suggest to surgeons that the optimal management of patients with lower gastrointestinal hemorrhage is that those patients with sources of bleeding that can be localized should undergo partial colectomy, whereas those in whom a specific source cannot be localized should undergo total colectomy [2]. The data utilized in this study do not include diagnosis of source of lower gastrointestinal hemorrhage in either surgical group. It was also noted that total abdominal colectomy had a significantly higher rate of mortality of $27 \%$ compared to partial colectomy which had a mortality of $10 \%$ [3]. Contrary to these findings, a study by Farner et al found in their retrospective study of 77 patients from their institution, that there was no significant difference in mortality between those patients who had undergone partial vs. total colectomy [4]. Renzulli et al similarly found no significant difference in postoperative morbidity or mortality when comparing patients who had undergone segmental and subtotal colectomy at their institution [5]. We found no significant difference in mortality between patients who had underwent total vs. partial colectomy.

We found that patients who underwent total abdominal colectomy were more likely to have greater than four blood transfusions prior to surgery than those who underwent partial colectomy, possibly secondary to delay in localization of source of bleeding. The data that we utilized for this study do not include amount of time from presentation to surgery. These findings are different than those found in a prior study by Renzulli et al comparing patients who had undergone segmental vs. subtotal colectomy in one institution. This group found no significant difference in transfusion prior to surgery between the two groups, though they did find an increase in amount of blood transfused intra-operatively in patients who had undergone subtotal colectomy compared to segmental colectomy [5]. The data set that we utilized represents amount of blood transfused only prior to surgery and does not supply any data regarding intraoperative blood transfusion or need for transfusion following surgery. An additional limitation of our data is that the database does not differentiate beyond greater or less than four units of blood transfused prior to surgery.

The NSQIP dataset does not elaborate on specific diagnosis or cause of bleeding or whether or not the source of bleeding was identified prior to surgery. Many of the publications that exist regarding management of lower gastrointestinal hemorrhage hinge their recommendations on the localization of hemorrhage. The general consensus of most published papers to date suggests that in patients for whom a specific location can be identified of hemorrhage, a partial colectomy is recommended. In patients where a specific source cannot be localized, total colectomy was the recommended operation.

\section{Conclusion}

The major finding of our review of a recent national cohort was

Table 2. Total vs. Partial Colectomy for Indication of Hemorrhage

\begin{tabular}{llll}
\hline & Total colectomy & Partial colectomy & P-value \\
\hline Receive $>4$ units of blood prior to surgery & $78 \%$ & $55 \%$ & $<0.01$ \\
Operative time $>180$ min & $43 \%$ & $23 \%$ & $<0.01$ \\
Laparoscopic procedure & $35 \%$ & $20 \%$ & 0.02 \\
Stoma at time of surgery & $21 \%$ & $2 \%$ & $<0.01$ \\
\hline
\end{tabular}


that the most common procedure performed for lower gastrointestinal hemorrhage was partial colectomy. Total colectomy was associated with an increased risk of cardiac and renal complications, but not with increased mortality. We are unable to make practice recommendations based on this information because of limitations of the retrospective database. These limitations include lack of information regarding specific diagnosis of source of hemorrhage and which diagnostic procedures had been performed prior to surgery.

\section{Disclosures}

Presented as an ePoster at ASCRS 2016 Meeting in Los Angeles, CA, April 30, 2016 to May 4, 2016. Presented as a poster at the Pennsylvania Society of Colon and Rectal Surgeons Resident Research Meeting on April 1, 2016. Accepted as a poster presentation at the 2016 ACS-NSQIP Conference in San Diego, CA, July 16, 2016 to July 19, 2016.

\section{Disclaimer}

None.

\section{Sources of Funding}

None.

\section{References}

1. American College of Surgeons National Surgical Quality Improvement Program. Chicago: ACS-NSQIP; ACSNSQIP Semiannual Report July 1, 2012, through 30 June 2013.

2. Schuetz A, Jauch KW. Lower gastrointestinal bleeding: therapeutic strategies, surgical techniques and results. Langenbecks Arch Surg. 2001;386(1):17-25.

3. Hoedema RE, Luchtefeld MA. The management of lower gastrointestinal hemorrhage. Dis Colon Rectum. 2005;48(11):2010-2024.

4. Farner R, Lichliter W, Kuhn J, Fisher T. Total colectomy versus limited colonic resection for acute lower gastrointestinal bleeding. Am J Surg. 1999;178(6):587-591.

5. Renzulli P, Maurer CA, Netzer P, Dinkel HP, Buchler MW. Subtotal colectomy with primary ileorectostomy is effective for unlocalized, diverticular hemorrhage. Langenbecks Arch Surg. 2002;387(2):67-71. 